\title{
Chromosomal segregation in spermatozoa of five Robertsonian translocation carriers $\mathbf{t}(13 ; 14)$
}

\author{
Manel Mahjoub • Meriem Mehdi • Sonia Brahem • \\ Hatem Elghezal $\cdot$ Samira Ibala $\cdot$ Ali Saad
}

Received: 21 December 2010 / Accepted: 18 March 2011/Published online: 30 March 2011

(C) Springer Science+Business Media, LLC 2011

\begin{abstract}
Purpose To analyse the segregation of a Robertsonian translocation $\mathrm{t}(13 ; 14)$ in five male carriers, and to verify a possible inter-chromosomal effect (ICE) of the Robertsonian translocation on chromosomes $18, \mathrm{X}$, and $\mathrm{Y}$.

Methods The spermatozoa of these patients $(n=5)$ and of 15 donors with normal semen parameters and 46,XY karyotype were analysed using triple colour FISH with locus specific probes for chromosomes 13, 14, and 21 and by triple colour FISH for chromosomes X, Y, and 18.

Results The frequency of balanced spermatozoa resulting from alternate segregation varied between $62.16 \%$ and $81.70 \%$ with a mean of $71.5 \%$. The rates of unbalanced spermatozoa resulting from adjacent segregation varied between $13.4 \%$ and $25.1 \%$ with a mean of $18.26 \%$. Triple colour FISH X-Y-18 showed a significant increase in disomy frequencies of these chromosomes in comparison with controls, indicating an ICE.

Conclusion In spite of the high number of normal/balanced frequencies, there remain many unbalanced spermatozoa resulting from adjacent mode of segregation. This raises the question of the unbalanced chromosomal risk for the offspring of $45, \mathrm{XY}, \mathrm{t}(13 ; 14)$ males and the importance of the genetic counselling prior to ICSI or IVF treatment for couples where the male is a Robertsonian translocation carrier.
\end{abstract}

Capsule Despite the high number of normal/balanced frequencies, there remain many unbalanced spermatozoa resulting from adjacent mode of segregation.

M. Mahjoub $\cdot$ M. Mehdi $\cdot$ S. Brahem $(\bowtie) \cdot H$. Elghezal

S. Ibala $\cdot$ A. Saad

Department of Cytogenetic and Reproductive biology, Farhat

Hached, University Teaching Hospital,

4000 Sousse, Tunisia

e-mail: brahemsonia@yahoo.fr
Keywords FISH $\cdot$ Meiotic segregation $\cdot$ Robertsonian translocation $\cdot$ Spermatozoa

\section{Introduction}

Robertsonian (Rob) translocations are one of the most common structural aberration observed, within an incidence of 1.23/1000 newborns [1]. Among them, the most frequently seen in the general population is $\mathrm{t}(13 ; 14)$, with an incidence of 0.97/1000 newborn carriers [1]. In general, carriers of Rob translocations are phenotypically normal but these men can have problems of infertility associated with more or less severe oligozoospermia. Furthermore, these carriers of Rob translocations are at increased risk for spontaneous abortions and chromosomally unbalanced offspring.

The infertility origin in these individuals has been directly related to the meiotic process. In Rob translocations pairing of the reorganized chromosomes during prophase I give rise to a trivalent structure [2]. It is well known that this meiotic configuration tends to segregate in alternate way [3], resulting in the production of normal or balanced spermatozoa. However a certain percentage of unbalanced spermatozoa deriving from adjacent segregations are also produced, and could be responsible for the miscarried or the severely affected aneuploidy offspring frequently born to these carriers [4]. Furthermore, the meiotic disturbances resulting from the behaviour of the reorganized chromosomes and of other bivalents could lead to different degrees of meiotic arrest, resulting in the oligozoospermia or azoospermia frequently observed in these patients. On the other hand, the possible occurrence of interchromosomal effects (ICE), affecting the normal disjunction of other chromosome pairs not involved in any reorganization, has been a classical topic of discussion. 
Several groups using different cytogenetic techniques of spermatozoa have tried to analyse the meiotic segregation of specific reorganizations and to evaluate the final production of balanced or unbalanced sperm, in order to offer patients accurate reproductive advice. Cytogenetic analysis of sperm nuclei using fluorescence in-situ hybridization (FISH) techniques with different chromosome specific-probes has proven to be a useful tool to determine the meiotic segregation especially in translocation carriers. Analysing cells by using FISH is less time consuming than the hamster ovum penetration test and karyotyping [5], and many cells can be examined in a relatively short period [611]. The aim of this study was to analyse using a triple colour FISH the meiotic segregation of a Rob translocation $\mathrm{t}(13 ; 14)$ in five male carriers and to verify a possible interchromosomal effect of the Rob translocation on chromosomes $18, \mathrm{X}$, and $\mathrm{Y}$.

\section{Materials and methods}

\section{Patients}

Five unrelated male carriers of the Rob translocation $t$ $(13 ; 14)$, consulting for infertility problem in our laboratory of Cytogenetic and Reproductive Biology, Farhat Hached University Teaching Hospital, were included in this study. In addition 15 fertile men with normal 46, XY karyotype and normal sperm characteristics were recruited as a control group for the analysis of the meiotic segregation of chromosomes 18, $\mathrm{X}$ and $\mathrm{Y}$.

This protocol was approved by the local ethics committee and all patients and controls had previously given informed consent for the study.

\section{Semen analysis}

Semen samples were produced by masturbation after 3 days of sexual abstinence from patients and controls. After liquefaction of semen, at room temperature, standard semen parameters were obtained according to World Health Organization (WHO) guidelines [12].

\section{Semen preparation}

After semen analysis, spermatozoa from fresh semen were washed twice in $8 \mathrm{ml}$ of Phosphate Buffered Saline (PBS, pH 7.4) (Sigma: St Louis, MO, USA) by centrifugation at $400 \mathrm{~g}$ for $5 \mathrm{~min}$. Then the final pellets were fixed with $5 \mathrm{ml}$ of acetic acid/methanol mixture (MERCK, Darmstadt, Allemande) for at least $30 \mathrm{~min}$ at $4^{\circ} \mathrm{C}$. Aliquots $(40$ $50 \mu \mathrm{l})$ of the resulting suspension of nuclei were smeared on slides. At least two slides were prepared for each patient and controls. These slides were kept frozen at $-20^{\circ} \mathrm{C}$ until use for FISH.

Aneuploidy analysis

In order to get the sperm chromatin accessible to DNA probes, slides were incubated in $\mathrm{NaOH} 1 \mathrm{~N}$, at room temperature for $2 \mathrm{~min}$. The slides were distilled water washed. Then, they were dehydrated through an ethanol series (70-90-100\%) and air-dried.

\section{DNA probes}

By using two-colour FISH, it was not possible to differentiate between gametes with 3:0 segregation and diploid spermatozoa (both cases show two hybridization signals for the probes used). So a triple-colour FISH analysis using locus specific probes for chromosomes 13 (LSI 13q12, spectrum green), 14 (LSI 14q11, spectrum red) and 21 (LSI 21q22, spectrum yellow) was carried out on spermatozoa of the Rob translocation carriers. A cocktail of these three probes was prepared.

In order to verify a possible inter-chromosomal effect of the Rob translocation on chromosomes X, Y, and 18, a triple colour FISH with specific alphoid probes of the $\mathrm{X}$ chromosome (probe DXZ1, spectrum red; Abbott), Y chromosome (probe DYZ3, spectrum yellow; Abbott), and 18 chromosome (D18Z1, spectrum green; Abbott) were applied in spermatozoa of patients and controls. A cocktail of these three probes was prepared.

The two mixtures of probes were denaturised at $72^{\circ} \mathrm{C}$ for 8 min and snap-cooled on ice.

\section{Fluorescence in situ hybridization}

The slides were incubated in a denaturation solution of $70 \%$ formamide, 20× saline sodium citrate (SSC) (pH 5,3) and distilled water at $72^{\circ} \mathrm{C}$ for $2 \mathrm{~min}$. Slides were snap-cooled in $70 \%$ ethanol at $-20^{\circ} \mathrm{C}$ for $2 \mathrm{~min}$ and then dehydrated through an ethanol series (90-100\%) at room temperature before the probe mixture was applied to the sperm slides. The slides were hybridized overnight for locus specific probes (chromosomes 13, 14 and 21) and for $2 \mathrm{~h}$ at $37^{\circ} \mathrm{C}$ for centromeric probes (chromosomes $\mathrm{X}, \mathrm{Y}$, and 18). Finally, the slides were washed in $1 \times \mathrm{SSC}$, counterstained and stored in the dark at $4^{\circ} \mathrm{C}$ prior to carrying out microscopic observation.

\section{Scoring criteria}

Scoring was performed on an Axioplan epifluorescence microscope with three filters for DAPI, Fluorescein Isothiocyanate (FITC) and Rhodamine (Leica, Wetzlar, 
Germany). Only intact spermatozoa bearing a similar degree of decondensation and clear hybridization signals were scored; disrupted or overlapping spermatozoa were excluded from analysis. Spermatozoa were regarded as disomic if they presented two distinct hybridization signals for the same chromosome, each equal in intensity and size to the single signal found in normal monosomic nuclei.

Only slides with hybridization rate $\geq 98 \%$ were analysed and at least 100 sperm nuclei per probe were scored.

\section{Statistical analysis}

Chi-squared test was used to compare the aneuploidy rates between patients and controls. A probability value of less than 0.05 was considered to be statistically significant.

\section{Results}

Sperm characteristics of patients carriers of Robertsonian translocation $\mathrm{t}(13 ; 14)$

The age of our patients ranged from 36 to 41 years with a mean of 39 years. The characteristics of sperm examination of patients and controls are given in Table 1. According to WHO Criteria, our patients had very poor semen parameters. Four patients showed an oligoasthenoteratozoospermia (OAT) and the last patient showed an asthenoteratozoospermia (AT). Only one patient showed a normal concentration and for the remaining the patient sperm count varied between $0.14 \times 10^{6}$ \% $\mathrm{ml}$ and $6.82 \times 10^{6} / \mathrm{ml}$.

\section{Segregation of Robertsonian translocation $\mathrm{t}(13 ; 14)$}

Results from three colour FISH 13-14-21 for the analysis of the chromosomes status in the sperm of the five Rob translocation carriers are given in Table 2 and illustrated in Fig. 1. Segregation analysis of the chromosomes involved was ascertained in a total of 842 spermatozoa, ranging from 149 to 185 cells evaluated.

The frequency of balanced spermatozoa resulting from alternate segregation varied between $62.16 \%$ and $81.70 \%$ with a mean of $71.5 \%$. Whereas, the rates of unbalanced spermatozoa, resulting from adjacent segregation, varied between $13.4 \%$ and $25.1 \%$ with a mean of $18.26 \%$.

Using a triple colour FISH makes it possible to differentiate between 3:0 or diploid cells. As shown in Table 2, the rate of gametes resulting from 3:0 segregation $(4.47 \%)$ was significantly higher than the rate of diploid gametes $(1.94 \%)(p<0.05)$.

The nullisomy and disomy rates for the chromosomes 13, 14 and 21 for each patient are reported in Table 3. There was an increased incidence of chromosomes 13, 14, and 21 disomy and nullisomy in these patients. However no statistical difference was found between the rate of disomic and nullisomic spermatozoa for each chromosome $(p>0.05)$.

Inter-chromosomal effect of the Rob translocation on chromosomes $\mathrm{X}, \mathrm{Y}$, and 18

A total of 2074 spermatozoa were analysed to evaluate the occurrence of ICE. Triple colour FISH X-Y-18 (Table 4) showed a significant increase in $\mathrm{XX}, \mathrm{YY}$, and chromosome 18 disomy frequencies compared to the controls $(p<0.05)$. However disomy XY was not significantly different between controls and patients $(p>0.05)$.

Aneuploidy of chromosome 18 presented an average frequency less raised with regard to the average frequency of the sex chromosome aneuploidy (3.95\% versus $6.64 \%$ ) in patients as well as in controls $(0.22 \%$ versus $1.16 \%)$.

FISH data from ICE studies were also used to evaluate the diploidy frequency. A statistical difference versus controls was noted $(p<0.05)$.

The frequency of sperm nuclei bearing $18 \mathrm{XX}$ or $18 \mathrm{YY}$ relative to the second meiosis abnormalities (MII) was significantly more important than the frequency of the nuclei sperm bearing $18 \mathrm{XY}$ relative to the first meiosis abnormalities (MI) $(4,89 \%$ versus $0,46 \% ; p<0.05)$.

\section{Discussion}

Multicolour FISH on interphase spermatozoa is the only means which allows a direct tool to analyze the meiosis
Table 1 Semen parameters of the five patients with Rob translocation $\mathrm{t}(13 ; 14)$ and controls

\begin{tabular}{lccccccc}
\hline Semen parameters & \multicolumn{3}{l}{ Patient number } & & & Control group \\
\cline { 2 - 7 } & 1 & 2 & 3 & 4 & 5 & Mean \pm SD & Mean \pm SD \\
\hline Volume (ml) & 3.8 & 3.5 & 2.3 & 4 & 2 & $3.12 \pm 0.90$ & $03.20 \pm 1.43$ \\
Sperm count $\left(\mathrm{X} 10^{6} / \mathrm{ml}\right)$ & 4.62 & 0.48 & 6.82 & 0.14 & 34.3 & $9.27 \pm 14.27$ & $139 \pm 59.95$ \\
Abnormal forms (\%) & 87 & 96 & 83 & 75 & 86 & $85.4 \pm 7.56$ & $54.33 \pm 10.65$ \\
Total motility (\%) & 30 & 20 & 50 & 30 & 30 & $32 \pm 10.95$ & $27.00 \pm 4.02$ \\
Multiple Anomalies Index & 1.9 & 1.58 & 1.9 & 1.74 & 1.87 & $1.79 \pm 0.13$ & $1.41 \pm 0.14$ \\
\hline
\end{tabular}


Table 2 Segregation analysis results in the five Robertsonian translocation carriers $\mathrm{t}(13 ; 14)$ analysed

\begin{tabular}{lcccccc}
\hline \multicolumn{7}{l}{ Patient number } \\
\cline { 2 - 7 } & 1 & 2 & 3 & 4 & 5 & Mean \pm DS \\
\hline No. of spermatozoa & 149 & 185 & 164 & 179 & 165 & $168.4 \pm 14.09$ \\
Alternate mode (\%) & 69.79 & 62.16 & 81.70 & 68.71 & 75.15 & $71.5 \pm 7.33$ \\
Adjacent mode (\%) & 20.1 & 15.68 & 13.4 & 25.1 & 17 & $18.26 \pm 4.52$ \\
3: 0 mode (\%) & 6.71 & 2.7 & 4.27 & 3.64 & 5.03 & $4.47 \pm 1.51$ \\
Diploidy (\%) & 3.36 & 1.7 & 0.61 & 2.23 & 1.82 & $1.94 \pm 0.99$ \\
\hline
\end{tabular}

progress in higher number of cells and to identify a possible chromosomally unbalanced offspring.

In the present study, the assessment of the meiotic segregation using a triple colour FISH 13-14-21, of five infertile men with Rob translocation $\mathrm{t}(13 ; 14)$ shows a homogeneous frequency of balanced spermatozoa resulting from alternate segregation which varies from $62.16 \%$ to $81.70 \%$. Our results are in agreement with the published data of eight previous reports which have shown that in general most of the spermatozoa in Rob translocation carriers $\mathrm{t}(13 ; 14)$ are balanced for the chromosomes involved in translocation with a prevalence of the alternate mode of segregation [9, 11, 13-15]. Three different techniques were used for the study of meiotic segregation in these different studies. The two first studies $[13,16]$ were performed by the human-hamster inter-specific fertilization system, the third one [17] was performed by the injection of human sperm into mouse oocytes and the rest of studies used the FISH technique [9, 11, 14, 15]. The rate of balanced gametes varied for the seven studied patients from $73.50 \%$ [16] to $92.30 \%$ [13]. All these studies have indicated a prevalence of the alternate mode of segregation. This alternate mode prevalence can be explained by meiotic

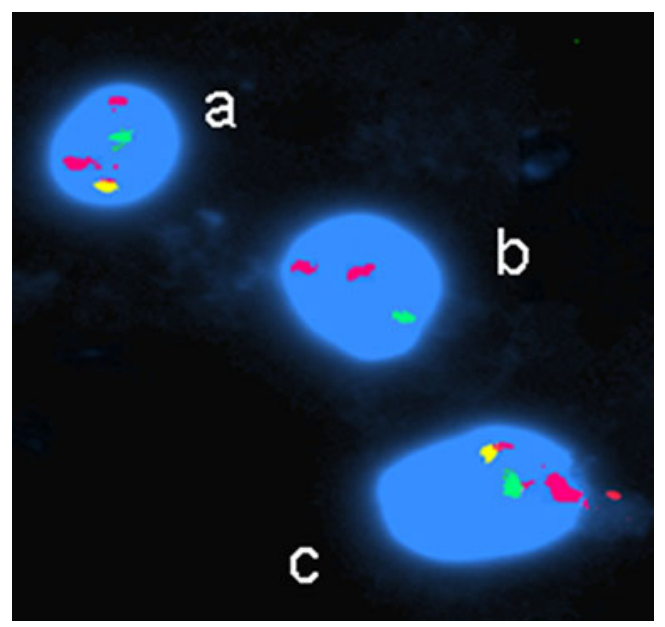

Fig. 1 Triple colour-FISH on decondensed sperm nuclei from a $t$ $(13 ; 14)$ carrier using locus-specific probes (13q12 spectrum green, $14 \mathrm{q} 11$ spectrum red, 21q22 spectrum yellow). a Chromosome 14 disomic sperm. b Chromosome 14 disomic and chromosome 21 nullosomic sperm. c Normal spermatozoa cytogenetic studies which indicate that the trivalent at pachytene stage is always in cis-configuration [18]. In spite of the high number of normal/balanced frequencies, there is still much unbalanced spermatozoa (mean: 18.26\%) in our study resulting from adjacent mode of segregation. In the literature, the rate of unbalanced gametes varied from $7.70 \%$ [13] to $26.50 \%$ [16]. This wider range reported in other studies and compared with our own may be related to technical aspects, such as the number of cells analysed (human-hamster fertilization system versus FISH), the characteristics and the combination of probes used (locusspecific, sub-telomeric or chromosomes paints) and the specific scoring criteria used.

Using a triple colour FISH 13-14-21, is possible to distinguish 3:0 mode of segregation from diploid gametes. In our study, for each patient the frequency of gametes resulting from 3:0 segregation was higher than diploid gametes. Our results are in discordance with the data of Ogur et al. [14] who showed that the majority of the cells are diploid.

Meiotic segregation analysis can predict gamete behaviour in patients with chromosomal abnormalities and can be useful for genetic counselling purposes. One of the remaining questions that we tend to answer is whether the meiotic association of translocated chromosomes could affect the meiotic behaviour of other chromosomes. For this raison, in our present work, we try to analyze the segregation pattern of the translocated chromosomes in spermatozoa of five $\mathrm{t}(13 ; 14)$ carriers then to evaluate a possible interchromosomal effects (ICE) for chromosomes $18, \mathrm{X}$ and $\mathrm{Y}$ in these carriers.

In Robertsonian translocations, the adjacent modes of segregation should theoretically involve the same rates of spermatozoa carrying a 13 or 14 supernumerary chromosome as that of nullisomic spermatozoa for the same chromosome. Our data are in close agreement with this theory. However Morel et al. [19] had found that the percentages of chromosomes 13 or 14 nullisomic spermatozoa were higher than those of chromosome 13 or 14 disomic gametes. Moreover, in our series, we had found a significant increase of disomy frequencies for chromosomes 21 (a mean: 7.74\%) and for chromosomes 18 compared to the controls (respective means: $2.24 \%$ versus $0.12 \% ; p<$ 
Table 3 Frequencies of spermatozoa considered to be disomic and nullisomic for chromosomes 13, 14 and 21 in the five Rob translocation carriers $\mathrm{t}(13 ; 14)$

\begin{tabular}{|c|c|c|c|c|c|c|}
\hline \multirow[t]{2}{*}{ Patient No. } & \multicolumn{2}{|c|}{ Chromosome 13} & \multicolumn{2}{|c|}{ Chromosome 14} & \multicolumn{2}{|c|}{ Chromosome 21} \\
\hline & Disomy (\%) & Nullisomy (\%) & Disomy (\%) & Nullisomy (\%) & Disomy (\%) & Nullisomy (\%) \\
\hline 1 & 8.05 & 9.4 & 8.72 & 7.38 & 10.07 & 8.05 \\
\hline 2 & 8.11 & 4.32 & 3.78 & 4.86 & 7.57 & 5.4 \\
\hline 3 & 7.93 & 4.87 & 6.1 & 3.05 & 5.5 & 6.1 \\
\hline 4 & 7.82 & 9.5 & 8.94 & 6.7 & 8.9 & 7.9 \\
\hline 5 & 8.48 & 7.27 & 5.45 & 7.88 & 6.66 & 4.2 \\
\hline Means & 8.08 & 7.07 & 6.6 & 6.06 & 7.74 & 6.33 \\
\hline
\end{tabular}

0.05). these findings would seem to indicate an interchromosomal effect. As suggested by Anton et al. (2004) [11], inter-individual variations concerning the presence or absence of an inter-chromosomal effect that are observed among the carriers can be related to specific characteristic of the translocated chromosomes, e.g. satellite polymorphisms common in acrocentric chromosomes.

In the ICE evaluation, our triple FISH $18-\mathrm{X}-\mathrm{Y}$ results showed a significant high mean rate of disomy sex chromosomes for our five $t(13 ; 14)$ carriers (a mean: $3.55 \%$ ). These findings are in close agreement with the results of Vegetti et al. [20] and Morel et al. [19] who had shown an increased frequency of sex chromosomes disomies in the spermatozoa of some of the $t(13 ; 14)$ carriers. Indeed, Luciani and Guichaoua [21] have developed a hypothesis that there is an interaction between the trivalent structure of Rob translocation and the sexual vesicle. They explained that the increase of the sex chromosomes disomy may be due to a possible influence of the translocated chromosomes on the synapsis and disjunction of sex chromosome pairs. However, the interchromosomal effect analysis of spermatozoa for patient 4 was not possible because of the extremely low sperm concentration $\left(0.14 \times 10^{6} / \mu \mathrm{l}\right)$ that could be related to aneuploidy and diploidy. Most carriers of Robertsonian translocations have oligozoospermia. This may be a result of the intervention of meiotic checkpoints during meiosis (erratic chromosomes, lack of tension), when the cell may be unable to complete the meiotic process that leads the cell into apoptosis. If the cell is capable of completing the division process, the result may be the production of aneuploid or diploid spermatozoa.

Our comparative study of meiotic segregation for sex chromosomes noted that the disomy XX and YY rates are more significant than the disomy $\mathrm{XY}$ rate in patients. It means that disomy due to the meiosis II abnormalities (XX or YY) is more important than disomy due to the meiosis I abnormalities (XY). These findings are in agreement with the previous reports $[11,14,19]$. The higher incidence of spermatozoa bearing disomies XX or YY indicates that the meiotic arrest has been specifically performed in meiosis II in some germ cells which results in the oligozoospermia and aneuploidy in other cells capable to complete spermatogenesis. Thus, the meiosis I checkpoint is insufficient to stop the division of germ cells carrying unbalanced chromosomal abnormalities.

Our data provides evidence of an ICE for sex chromosomes in agreement with Anton et al. (2004) study [11]. They observed high sex chromosome aneuploidy rates $(0.68 \%)$ in the sperm of Robertsonian translocation carriers compared to controls $(0.37 \%)$ and insignificant rates for other chromosomes. Some investigations have shown an ICE [22, 23], but
Table 4 Results of triple colour FISH X-Y-18 performed on spermatozoa of the five Rob translocation carriers $\mathrm{t}(13 ; 14)$ and controls

\begin{tabular}{|c|c|c|c|c|c|c|c|}
\hline & \multicolumn{3}{|c|}{ Chromosome 18} & \multicolumn{3}{|c|}{ Sex chromosomes } & \multirow[t]{2}{*}{ Diploidy } \\
\hline & Nullisomy & Disomy & Nullisomy & Disomy X & Disomy Y & Disomy XY & \\
\hline Patient 1 & 1.18 & 2.17 & 0.2 & 3.6 & 1.78 & 0.2 & 0.2 \\
\hline Patient 2 & 2.56 & 3.2 & 3.2 & 5.76 & 1.28 & 0.64 & 0.64 \\
\hline Patient 3 & 1.18 & 2.24 & 1.49 & 2.62 & 1.96 & 0.65 & 1.09 \\
\hline Patient 4 & 2.57 & 1.54 & 1.54 & 4.12 & 0 & 0 & 1.54 \\
\hline Patient 5 & 1.07 & 2.06 & 0 & 1.67 & 1.67 & 0.83 & 0.26 \\
\hline Means & 1.71 & 2.24 & 1.28 & 3.55 & 1.33 & 0.46 & 0.74 \\
\hline Controls & 0.1 & 0.12 & 0.18 & 0.28 & 0.28 & 0.42 & 0.16 \\
\hline
\end{tabular}


others either did not show it $[7,24,25]$ or attributed the increased aneuploidy rate due to the oligoasthenoteratozoospermia (OAT) phenotype frequency observed within these patients $[10,26,27]$ or only to the ICE. For this reason, other FISH studies on spermatozoa from a large number of Robertsonian, translocations carriers should be performed in order to give precise information about the genetic risks linked to those who carry structural chromosomes abnormalities. The analysis of the meiotic segregation of chromosomes involved in structural chromosomes rearrangement and ICE can generate a more personalised risk assessment for the reproductive outcome of patients with structural chromosome aberrations that can be a useful in genetic counselling.

In the reviews of literature, the segregation studies performed by FISH on spermatozoa from males whom are fertile and carriers of structural chromosomal abnormalities as Robertsonian translocations are not mentioned. There is no investigation which studied the meiotic segregation of fertile male Robertsonian translocation $\mathrm{t}(13 ; 14)$ and which is compared with those of infertile male $t(13 ; 14)$ carriers. In the literature, the semengram of Robertsonian translocation carriers have not shown normal semen parameters because Robertsonian translocation carriers produce a large number of chromosomally unbalanced gametes.

Finally, we can conclude that $\mathrm{t}(13 ; 14)$ carriers have an homogeneous segregation pattern with a clearly preferential alternate segregation. However, in spite of the high number of normal/balanced frequencies, there is still much unbalanced spermatozoa resulting from adjacent mode of segregation. Also we had shown an ICE on chromosomes $\mathrm{X}, \mathrm{Y}, 18$, and 21.

\section{References}

1. Nielsen J, Wohlert M. Chromosome abnormalities found among 34910 newborn children: results from 13 -year incidence study in Arhus, Denmark. Hum Genet. 1991;87:81-3.

2. Vidal F, Templado C, Navarro J, Marina S, Egoscue J. Meiotic and synaptonemal complexes studies in a 14/21 translocation carrier. Int J Androl. 1982;5:21-6.

3. Sybenga J. Chromosome structural variants. In: Sybenga J, editor. General cytogenetics. Amsterdam: North-Holland; 1975. p. 165-212.

4. Egoscue S, Blanco J, Vendrell JM, Garcia F, Veiga A, Aran B, et al. Human male infertility: chromosome anomalies, meiotic desorders, abnormal spermatozoa and recurrent abortion. Hum Reprod Update. 2000;6:93-105.

5. Balkan W, Martin RH. Segregation of chromosomes into the spermatozoa of a man heterozygeous for a 14;21 Robertsonian translocation. Am J Med Genet. 1983;16:169-72.

6. Rousseaux S, Hazzouri M, Pelletier R, Monteil M, Usson Y, Sèle B. Disomy rates for chromosomes 14 and 21 studied by fluorescent in-situ hybridization in spermatozoa from three men over 60 years of age. Mol Hum Reprod. 1998;4:695-9.
7. Honda H, Miharu N, Ohashi Y, Honda N, Hara T, Ohama K. Analysis of segregation and aneuploidy in two reciprocal translocation carriers, $t(3 ; 9)(\mathrm{q} 26.2 ; \mathrm{q} 32)$ and $t(3 ; 9)(\mathrm{p} 25 ; \mathrm{q} 32)$, by triple-color fluorescence in situ hybridization. Hum Genet. 1999;105:428-36.

8. Honda H, Miharu N, Samura O, He H, Ohama K. Meiotic segregation analysis of a 14;21 Robertsonian translocation carrier by fluorescence in situ hybridization. Hum Genet. 2000;106:18893.

9. Frydman N, Romana S, Le Lorc'h M, Vekemans M, Frydman R, Tachjian G. Assisting reproduction of infertile men carrying a Robertsonian translocation. Hum Reprod. 2001;16: 2274-7.

10. Moosani N, Pattinson HA, Carter MD, Cox DM, Rademaker AW, Martin RH. Chromosomal analysis of sperm from men with idiopathic infertility using sperm karyotyping and fluorescence in situ hybridization. Fertil Steril. 1995;64:811-7.

11. Anton E, Blanco J, Egoscue J, Vidal F. Sperm FISH studies in seven male carriers of Robertsonian translocation $\mathrm{t}(13 ; 14)(\mathrm{q} 10$; q10). Hum Reprod. 2004;19:1345-51.

12. World Health Organization. Laboratory manual for the examination of human semen and sperm-cervical mucus interaction. New York: Cambridge University Press; 1999.

13. Pelloster F, Sèle B, Jalbert H. Chromosome analysis of spermatozoa from a male heterozygous for a 13;14 Robertsonian translocation. Hum Genet. 1987;85:116-20.

14. Ogur G, Van Assche E, Vegetti W, Verheyen G, Tournaye H, Bonduelle $\mathrm{M}$, et al. Chromosomal segregation in spermatozoa of 14 Robertsonian translocation carriers. Mol Hum Reprod. 2006;12:209-15.

15. Escudero T, Lee M, Carrel D, Blanco J, Munne S. Analysis of chromosomes abnormalities in sperm and embryos from two 45, XY, t(13;14)(q10;q10) carriers. Prenat Diagn. 2000;20:599-602.

16. Martin RH. Cytogenetic analysis of sperm from a male heterozygous for a 13;14 Robertsonian translocation. Hum Genet. 1988;80:357-61.

17. Ogawa S, Araki S, Araki Y, Ohno M, Sato I. Chromosome analysis of human spermatozoa from an oligoasthenozoospermic carrier for a 13;14 Robertsonian translocation by their injection into mouse oocytes. Hum Reprod. 2000;15:1136-9.

18. Luciani JM, Guichaoua MR, Mattei A, Morazzani MR. Pachytene analysis of a man with a $13 \mathrm{q} ; 14 \mathrm{q}$ translocation and infertility. Behavior of the trivalent and nonrandom association with the sex vesicle. Cytogenet Cell Genet. 1984;38:14-22.

19. Morel F, Roux C, Bresson JL. FISH analysis of the chromosomal status of spermatozoa from three men with $45, \mathrm{XY}$, der $(13 ; 14)$ (q10;q10). Mol Hum Reprod. 2001;7:483-8.

20. Vegetti W, Van Asseche E, Frias A, Verheven G, Bianchi MM, Bonduelle $\mathrm{M}$, et al. Correlation between semen parameters and sperm aneuploidy rates investigated by fluorescence in-situ hybridization in infertile men. Hum Reprod. 2000;15:351-65.

21. Luciani JM, Guichaoua MR. L'incidence des anomalies chromosomiques de structure sur la spermatogenèse chez l'homme. Reprod Nutr Dev. 1990;1:95-103.

22. Vendrell JM, Garcia F, Veiga A, Calderon G, Egozcue S, Egozcue $\mathrm{J}$, et al. Meiotic abnormalities and spermatogenic parameters in severe oligoasthenozoospermia. Hum Reprod. 1999;14:375-8.

23. Blanco J, Egoscue J, Vidal F. interchromosomal effects for chromosome 21 in carriers of structural chromosome reorganizations determined by fluorescence in situ hybridization on sperm nuclei. Hum Genet. 2000;106:500-5.

24. Guttenbach M, Martinez-Exposito MJ, Michelmann HW, Engel W, Schmid M. Incidence of diploid and disomic sperm nuclei in 45 infertile men. Hum Reprod. 1997;12:468-73. 
25. Estop AM, Cieply K, Munne S, Surti U, Wakim A, Feingold E. Is there an interchromosomal effect in reciprocal translocation carriers? Sperm FISH studies. Hum Genet. 2000;106:51724.

26. Pang MG, Hoegerman SF, Cuticchia AJ, Moon SY, Doncel GF, Acosta AA, et al. Detection of aneuploidy for chromosomes 4, $6,7,8,9,10,11,12,13,17,18,21, \mathrm{X}$ and $\mathrm{Y}$ by fluorescence in-situ hybridization in spermatozoa from nine patients with oligoasthenoteratozoospermia undergoing intracytoplasmic sperm injection. Hum Reprod. 1999;14:1266-73.

27. Rives N, Saint Clair A, Mazurier S, Sibert L, Simeon N, Joly G, et al. Relationship between clinical phenotype, semen parameters and aneuploidy frequency in sperm nuclei of 50 infertile males. Hum Genet. 1999;105:266-72. 